\title{
Light Intensity on Intensive Care Units - A Short Review
}

\section{Critical Care and Circadian Rhythm}

Circadian rhythms are autonomous, self-sustained, approximately $24 \mathrm{~h}$ oscillations in biological processes (e.g. daily fluctuation of core body temperature, serum melatonin, or cortisol) entrained to environmental cues, the most important being light.

It is well recognised that the critical care environment is disruptive to maintenance of circadian rhythm and sleep-wake cycle. The normal rhythmic $24 \mathrm{~h}$ profiles of physiological parameters are altered in critical care patients [1-5]. This is especially seen in septic patients [6-8]. Mechanical ventilation, sedation, severity of illness and the ICU environment (noise) may all cause circadian disruption. However, it is now clear that light is the most powerful environmental influence on the circadian clock [9] (Figure 1).

\section{Sleep Deprivation on ICU}

As many as $61 \%$ of patients on critical care report sleep deprivation, placing it among the most common stressors experienced during critical illness [10]. Sleep deprivation and the inability to sleep are described by survivors as major sources of anxiety and stress during stays in the ICU [10-14]. Impaired circadian rhythm of melatonin secretion has been reported in sedated and mechanically ventilated patients in ICU [15]. It has been suggested that a reduction of plasma melatonin levels associated with the loss of circadian rhythms in critically ill patients receiving mechanical ventilation may contribute to sleep deprivation [15-19]. In addition to causing emotional distress, sleep deprivation in the critically ill has been hypothesised to contribute to critical care delirium, neurocognitive dysfunction, prolongation of mechanical ventilation and decreased immune function [20]. Polysomnography studies in both ventilated and non-ventilated critical care patients demonstrate that sleep disturbance is characterised by severe fragmentation and frequent arousals and awakenings. Critical care patient's sleep traverses the day-night interface, with approximately half or total sleep time occurring in the daytime $[20,21]$.

\section{Light and Circadian Rhythm}

Light is the most important 'resetter' of the circadian clock. Circadian rhythms are generated in a 'central' clock in the suprachiasmatic nucleus ( $\mathrm{SCN}$ ) of the mammalian brain. Light enters through the retina of the eye and stimulates the SCN through a non-visual pathway. Through this pathway light signals

\section{Hannah Jane Durrington}

Faculty of Biology, Division of Infection, Immunity and Respiratory Medicine, School of Biological Sciences, Medicine and Health, University of Manchester, Manchester, UK

\section{Corresponding author: \\ Hannah Jane Durrington}

\section{Hannah.durrington@manchester.ac.uk}

Faculty of Biology, Division of Infection, Immunity and Respiratory Medicine, School of Biological Sciences, Medicine and Health, University of Manchester, Manchester, UK.

\section{Tel: 04407980575901}

Citation: Durrington HJ. Light Intensity on Intensive Care Units - A Short Review. J Intensive \& Crit Care 2017, 3:2.

entrain the central circadian clock which in turn provides a source of timing information to the rest of the body.

\section{Disrupted Light Exposure on ICU}

There is disruption of the usual day/night pattern of light exposure when a patient is cared for on ICU. Patients are exposed to $24 \mathrm{~h}$ nursing care with un-naturally low light levels during the day and constant light interruptions through the night [22].

There is abundant evidence that disrupted light exposure can have adverse health effects. Several lines of research, ranging from human epidemiology to laboratory studies using animal models, indicate that disruption of a regular $24 \mathrm{~h}$ light-dark cycle increases morbidity and mortality [23-28].

Approximately $15-20 \%$ of workers in Europe and the United States participate in shift work [25]. Night shifts involve being in the presence of artificial light at night. This disturbance in the 'natural' daily light/dark cycle is responsible for an impaired circadian oscillator [29]. Epidemiological studies of shift-workers have demonstrated increased risks of breast, prostate, colorectal and endometrial cancers [30-33].

Jet Lag Disorder is generated by rapid travel across multiple time zones, a change too drastic to allow the circadian system to adapt smoothly. The most common jet lag symptoms include sleep impairment, rhythm desynchronisation, anxiety and depressed 


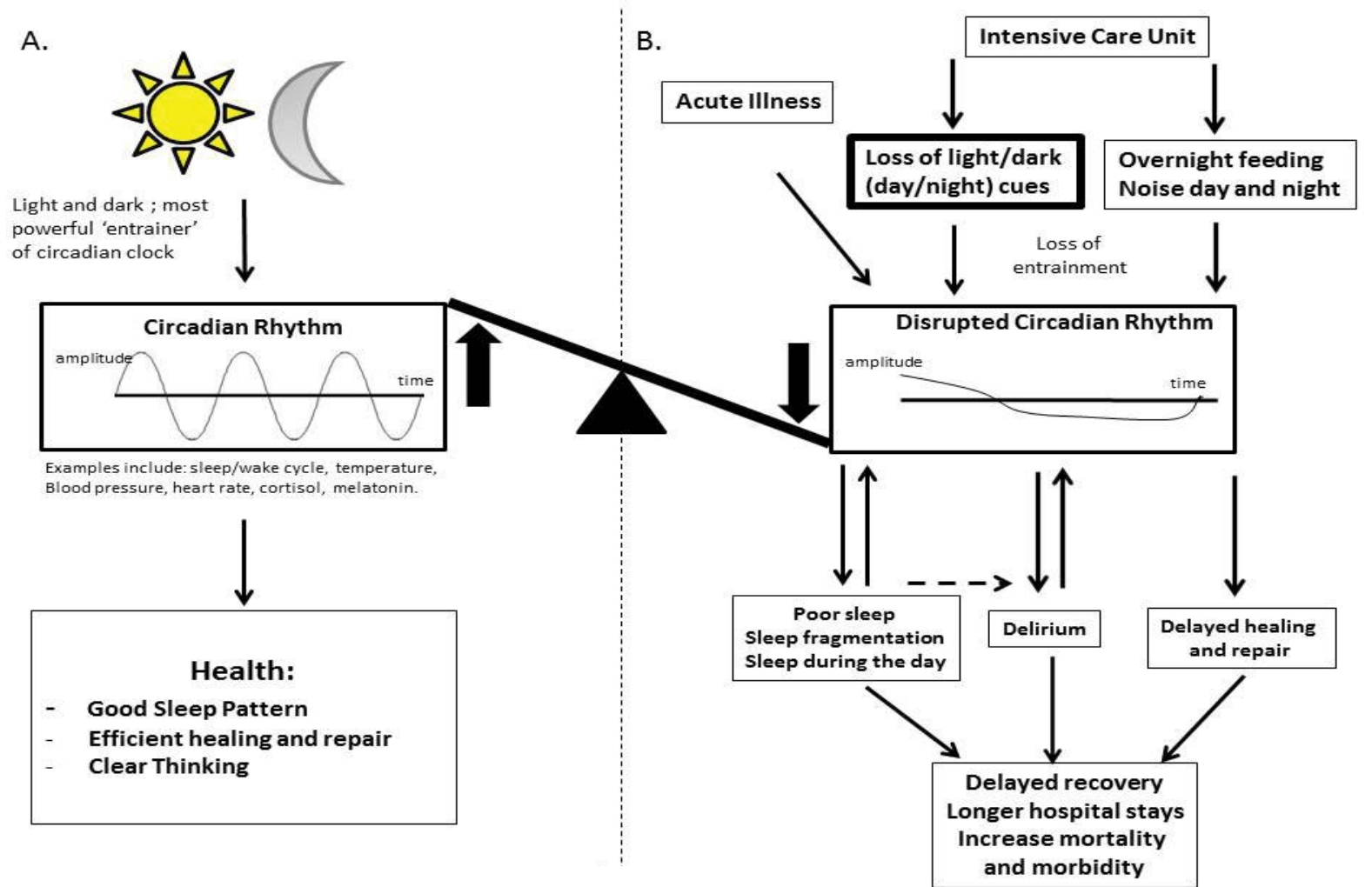

Figure 1 Light is the most important entrainer of circadian rhythm. In health, a large number of biological processes are under circadian control, leading to optimal sleep patterns, rapid healing and recovery and clear thinking. In the ICU environment many environmental cues are lost (the most important being day/night light changes) and this may result in disrupted circadian rhythms, leading to fragmented sleep patterns, delayed healing and recovery and delirium.

mood, gastrointestinal and cardiovascular complaints, dizziness and menstrual irregularity in women [34].

There is $50 \%$ mortality in rats recovering from sepsis if they are kept in constant dark or constant light conditions compared to rats exposed to day and night conditions (5-10\% mortality) [35]. Indicating the importance of maintaining day: night light patterns during the treatment of septic patients.

\section{Could Light Therapy Improve Clinical Outcome on ICU?}

The human retina contains a specialised photoreceptor distinct from the rods and cones and tasked with measuring ambient light levels. The information produced by this photoreceptor is sent directly to the hypothalamus, a part of the brain responsible for setting behavioural and physiological state. Via this route light influences many aspects of human biology including setting circadian clocks and inhibiting sleep [35]. The light environment of the ICU is highly un-natural. Light levels are generally subdued in the day compared to daylight and much higher through the night than most people would choose when at home [22]. Based upon experiments in animals we would expect this lack of a clear light: dark cycle to reduce the amplitude of circadian rhythms and, via this and direct effects of light at night, to impair sleep.
Fortunately, it is relatively straightforward to restore a more normal pattern of light exposure to patients in ICU. Through the evening and into the night eye masks can be worn to shield subjects from the lighting required to safely navigate the unit and to undertake clinical examinations. Similarly, a number of simple practical steps (upgrade room or bed lighting, orient patients towards windows, employ existing task lighting) could be used to reproduce a more normal daytime level of light exposure. The therapeutic administration of morning bright light has been used for decades in psychiatry to treat seasonal affective disorder [36]. Evidence from neonates suggests that cycled light improves sleep, alertness during the day, overall well-being and shortens time until discharge [37]. Furthermore, studies have reported reduced mortality in patients with myocardial infarction and less delirium and post-operative pain in critically ill surgical patients after exposure to bright natural light or windows [38,39]. A longitudinal study in critical care implementing nonpharmacological environmental changes designed to reduce disturbing patients during the night (noise and light reduction by use of blackout masks) demonstrated an impressive reduction in delirium and an improvement in sleep [40]. However, a recent clinical trial of continuous bright light therapy during the daytime in ICU (maximum light intensity 700 lux) concluded that there was no improvement in clinical outcome [41]. However, the maximal 
light intensity they achieved for the intervention was 700 lux (substantially below daylight levels) which may not have been a sufficiently large increase to observe an effect. For bright light therapy to be effective in entraining circadian rhythm, it does not need to be continuous; in fact, exposure to three consecutive bright light 'pulses' for just 15 min can be more effective than continuous bright light [42]. This may also be more practical for use on ICU. Interestingly, despite the fact that many patients on ICU have their eyes closed, it is well recognised that bright light can entrain circadian rhythm through non-visual pathways via the retina [9]. Bright light at night can shift the phase of circadian rhythms and reduce their amplitude [43]. Such high-intensity light interruptions are necessary for the $24 \mathrm{~h}$ care given to critically ill patients; however, wearing a black out mask overnight might minimise this potential disruption to circadian rhythm $[44,45]$.

\section{Future Studies}

We do not know what impact the loss of light cues has on disrupting circadian rhythm per se, compared to other environmental influences. Noise from monitors, drug-effects, mechanical ventilation, overnight naso-gastric feeding etc. mean that other circadian cues are also lost. However, given that light is the most powerful 'resetter' of the circadian clock, one would predict that it has a significant impact. It remains unclear if the circadian rhythm alterations observed in critically ill patients represent a compensatory response or whether they are in and of themselves pathologic. Prospective trials are needed to determine whether restoring sleep/circadian rhythms can influence clinical outcome or quality of life. With no clear guidelines as to what daytime light levels should be on ICU, a definitive clinical trial of bright light therapy (>1000 lux) during the day and black out masks at night is required to determine if there is a positive clinical outcome and also if circadian rhythms are re-established. Thus, the critical care community, itself subjected to frequent sleep disruption, has a unique opportunity to further explore the evolving field of chronobiology and therapeutics.

\section{Declaration}

HD is supported by Asthma UK, The Moulton Charitable Trust and the North West Lung Centre Charity. 


\section{References}

1 Tweedie IE, Bell CF, Clegg A, Campbell IT, Minors DS, et al. (1989) Retrospective study of temperature rhythms of intensive care patients. Crit Care Med 17: 1159-1165.

2 Olofsson K, Alling C, Lundberg D, Malmros C (2004) Abolished circadian rhythm of melatonin secretion in sedated and artificially ventilated intensive care patients. Acta Anaesthesiol Scand 48: 679-684.

3 Frisk U, Olsson J, Nylén P, Hahn RG (2004) Low melatonin excretion during mechanical ventilation in the intensive care unit. Clin Sci (Lond) 107: 47-53.

4 Pina G, Brun J, Tissot S, Claustrat B (2010) Long-term alteration of daily melatonin, 6-sulfatoxymelatonin, cortisol and temperature profiles in burn patients: A preliminary report. Chronobiol Int 27: 378-392.

5 Gazendam JA, Van Dongen HP, Grant DA, Freedman NS, Zwaveling JH, et al. (2013) Altered circadian rhythmicity in patients in the ICU. Chest 144: 483-489.

6 Mundigler G, Delle-Karth G, Koreny M Zehetgruber M, SteindlMunda P, et al. (2002) Impaired circadian rhythm of melatonin secretion in sedated critically ill patients with severe sepsis. Crit Care Med 30: 536-540.

7 Bagci S, Yildizdas D, Horoz OO, Reinsberg J, Bartmann P, et al. (2011) Use of nocturnal melatonin concentration and 6-sulfatoxymelatonin excretion to evaluate melatonin status in children with severe sepsis. J Pediatr Endocrinol Metab 24: 1025-1030.

8 Li CX, Liang DD, Xie GH, Cheng BL, Chen QX, et al. (2013) Altered melatonin secretion and circadian gene expression with increased proinflammatory cytokine expression in earlystage sepsis patients. Mol Med Rep 7: 1117-1122.

9 Lucas RJ, Peirson SN, Berson DM, Brown TM, Cooper HM, et al. (1972) Measuring and using light in the melanopsin age. Trends Neurosci 37: 1-9.

10 Wilson LM (1972) Intensive care delirium. The effect of outside deprivation in a windowless unit. Arch Intern Med 130: 225-226.

11 Simini B (1999) Patients' perceptions of intensive care. Lancet 354: 571-572.

12 Toublanc B, Rose D, Glerant JC, Francois G, Mayeux I, et al. (2007) Assist-control ventilation vs. low levels of pressure support ventilation on sleep quality in intubated ICU patients. Intensive Care Med 33: 1148-1154.

13 Davydow DS, Gifford JM, Desai SV, Bienvenu OJ, Needham DM (2009) Depression in general intensive care unit survivors: A systematic review. Intensive Care Med 35: 796-809.

14 Rotondi AJ, Chelluri L, Sirio C, Mendelsohn A, Schulz R, et al. (2002) Patients' recollections of stressful experiences while receiving prolonged mechanical ventilation in an intensive care unit. Crit Care Med 30: 746-752

15 Alexopoulou C, Kondili E, Vakouti E, Klimathianaki M, Prinianakis G, et al. (2007) Sleep during proportional-assist ventilation with load-adjustable gain factors in critically ill patients. Intensive Care Med 33: 1139-1147.

16 Olofsson K, Alling C, Lundberg D, Malmros C (2004) Abolished circadian rhythm of melatonin secretion in sedated and artificially ventilated intensive care patients. Acta Anaesthesiol Scand 48: 679-684.

17 Frisk U, Olsson J, Nylén P, Hahn RG (2004) Low melatonin excretion during mechanical ventilation in the intensive care unit. Clin Sci (Lond) 107: 47-53.

18 Shilo L, Dagan Y, Smorjik Y, Weinberg U, Dolev S, et al. (1999) Patients in the intensive care unit suffers from severe lack of sleep associated with loss of normal melatonin secretion pattern. Am J Med Sci 317: 278-281.

19 Ibrahim MG, Bellomo R, Hart GK, Norman TR, Goldsmith D, et al. (2006) A double-blind placebo-controlled randomised pilot study of nocturnal melatonin in tracheostomised patients. Crit Care Resusc 8: 187-191.

20 Paul T, Lemmer B (2007) Disturbance of circadian rhythms in analgosedated intensive care unit patients with and without craniocerebral injury. Chronobiol Int 24: 45-6134.

21 Weinhouse GL, Schwab RJ (2006) Sleep in the critically ill patient. Sleep 29: 707-716.

22 Parthasarathy S, Tobin MJ (2004) Sleep in the intensive care unit. Intensive care med 30: 197-206.

23 Blask DE, Dauchy R, Sauer L (2005) Putting cancer to sleep at night: The neuroendocrine/circadian melatonin signal. Endocrine 27: 179-188.

24 Durrington HJ, Clark R, Greer R, Martial FP, Blaikley J, et al. (2017) 'In a dark place, we find ourselves': Light intensity in critical care units. Int Care Med Experimental 5: 9.

25 Filipski E, Delaunay F, King VM, Wu MW, Claustrat B, et al. (2004) Effects of chronic jet lag on tumor progression in mice. Cancer Res 64: 7879-7885.

26 Stevens RG, Blask DE, Brainard GC, et al. Meeting report: the role of environmental lighting and circadian disruption in cancer and other diseases. Environ Health Perspect 2007; 115, 1357-62

27 Straif K, Baan R, Grosse Y, Secretan B, El Ghissassi F, et al. (2007) Carcinogenicity of shift-work, painting, and firefighting. Lancet Oncol 8: 1065-1066.

28 Kamdar BB1, Tergas Al, Mateen FJ, Bhayani NH, Oh J (2013) Night-shift work and risk of breast cancer: a systematic review and meta-analysis. Breast Cancer Res Treat 138: 291-301.

29 27. Pesch B, Harth V, Rabstein S, Johnni H, Steven WL, et al. (2010) Night work and breast cancer-results from the German GENICA study. Scand J Work Environ Health 36: 134-141.

30 Pronk A, Ji BT, Shu XO, Xue S, Yang G, et al. (2010) Night-shift work and breast cancer risk in a cohort of Chinese women. Am J Epidemiol 171: 953-959.

31 Erren TC, Reiter RJ (2009) Defining chronodisruption. J Pineal Res 46: 245-247.

32 Davis S, Mirick DK, Stevens RG (2001) Night shift work, light at night and risk of breast cancer. J Natl Cancer Inst 93: 1557-1562.

33 Erren TC, Reiter RJ (2008) A generalized theory of carcinogenesis due to chronodisruption. Neuro Endocrinol Lett 29: 815-821.

34 Schernhammer ES, Laden F, Speizer FE, Willett WC, Hunter DJ, et al. (2003) Night-shift work and risk of colorectal cancer in the nurse's health study. J Natl Cancer Inst 95: 825-828. 
35 Viswanathan AN, Hankinson SE, Schernhammer ES (2007) Night shift work and the risk of endometrial cancer. Cancer Res 67: 1061810622.

36 Brown GM, Pandi-Perumal SR, Trakht I, Cardinali DP (2009) Melatonin and its relevance to jet lag. Travel Med Infect Dis 7: 69-81.

37 Carlson D, Chiu W (2008) The absence of circadian cues during recovery from sepsis modifies pituitary-adrenocortical function and impairs survival. Shock 29: 127-132.

38 Rosenthal NE, Sack DA, Gillin JC, Lewy AJ, Goodwin FK, et al. (1984) Seasonal Affective Disorder. A description of the syndrome and preliminary findings with light therapy. Arch Gen Psychiatry 41: 72-80.

39 Morag I, Ohlsson A (2013) Cycled light in the intensive care unit for preterm and low birth weight infants. Cochrane Database Syst Rev CD006982.

40 Beauchemin KM, Hays P (1998) Dying in the dark: Sunshine, gender and outcomes in myocardial infarction. J R Soc Med 91: 352-354.
41 Walch JM, Rabin BS, Day R, Williams JN, Choi K, et al. (2005) The effect of sunlight on postoperative analgesic medication use: A prospective study of patients undergoing spinal surgery. Psychosom Med 67: 156-163.

42 Patel J, Baldwin J, Bunting P, Laha S (2014) The effect of a multicomponent multidisciplinary bundle of interventions on sleep and delirium in medical and surgical intensive care patients. Anaesthesia 69: 540-549.

43 Simons SK, Laheij RJF, van den Boogaard M, Moviat MAM, Paling $\mathrm{AJ}$, et al. (2016) Dynamic light application therapy to reduce the incidence and duration of delirium in intensive-care patients: A randomised controlled trial. Lancet Respir Med 4: 194-202.

44 Lucas RJ, Freedman MS, Muñoz M, Garcia-Fernández J-M, Foster RG (1999) Regulation of the mammalian pineal by non-rod, non-cone, ocular photoreceptors. Science 284: 505-507.

45 Dunlap JC, Loros JJ, DeCoursey PJ (2011) Chronobiology. Sinauer Associates Inc., USA. 